\title{
Integrated Scheduling of Production and Distribution with Release Dates and Capacitated Deliveries
}

\author{
Xueling Zhong' and Dakui Jiang ${ }^{2}$ \\ ${ }^{1}$ Department of Internet Finance and Information Engineering, Guangdong University of Finance, Guangzhou 510520, China \\ ${ }^{2}$ College of Management and Economics, Tianjin University, Tianjin 300072, China \\ Correspondence should be addressed to Xueling Zhong; zhongxuel@hotmail.com
}

Received 17 November 2015; Accepted 13 March 2016

Academic Editor: Alexandre B. Dolgui

Copyright (c) $2016 \mathrm{X}$. Zhong and D. Jiang. This is an open access article distributed under the Creative Commons Attribution License, which permits unrestricted use, distribution, and reproduction in any medium, provided the original work is properly cited.

This paper investigates an integrated scheduling of production and distribution model in a supply chain consisting of a single machine, a customer, and a sufficient number of homogeneous capacitated vehicles. In this model, the customer places a set of orders, each of which has a given release date. All orders are first processed nonpreemptively on the machine and then batch delivered to the customer. Two variations of the model with different objective functions are studied: one is to minimize the arrival time of the last order plus total distribution cost and the other is to minimize total arrival time of the orders plus total distribution cost. For the former one, we provide a polynomial-time exact algorithm. For the latter one, due to its NP-hard property, we provide a heuristic with a worst-case ratio bound of 2 .

\section{Introduction}

Integrated scheduling of production and distribution problem coordinates the scheduling, batching, and delivery decisions at the detailed scheduling level of the supply chain so as to achieve optimal operational performance in a supply chain. The problem has been attracting considerable attention from scheduling researchers as well as operation managers in the past few decades. In this paper, we study a static offline model on scheduling a set of $n$ orders, $N=\{1,2, \ldots, n\}$, on a single machine and batch delivering them to the customer. In the production stage, each order $j \in N$, associated with a release date $r_{j}$ and a processing time $p_{j}$, needs to be processed once without interruption. Order $j \in N$ cannot be processed before $r_{j}$ and is required to process $p_{j}$ time units on the machine. In the distribution stage, completed orders are delivered to the customer in batches by a sufficient number of homogeneous vehicles so that each vehicle will be used at most once and each delivery shipment will be transported by a dedicated vehicle. Without loss of generality, we assume that $n$ vehicles $\left(B_{1}, B_{2}, \ldots, B_{n}\right)$ are available at time 0 . Each order is packaged into a standard-size pallet for delivery convenience regardless of its size, and each vehicle can deliver at most $b$ orders. The delivery cost incurred by each shipment is $f$. Since all orders are delivered to the same customer and the delivery time of any vehicle is the same, we assume without loss of generality that the delivery time is 0 . For a given solution, we use $C_{j}, D_{j}$, and $T$ to denote the processing completion time of order $j \in N$, the arrival time of order $j \in N$, and total distribution cost, respectively. Additionally, define $D_{\max }=\max \left\{D_{j} \mid j \in N\right\}$ and $D_{\text {total }}=\sum_{j \in N} D_{j}$. The problem is to find a feasible solution such that one of the following two objective functions is minimized: (1) the arrival time of the last order plus total distribution cost, that is, $D_{\max }+T$; (2) the total arrival time of orders plus total distribution cost, that is, $D_{\text {total }}+T$. In the following, let $P 1$ and $P 2$ represent the two variations of model to minimize (1) and (2), respectively.

The researches on integrated scheduling of production and distribution models have been studied extensively in recent years, and the majority of them assume that all orders are available at time 0 (e.g., Hall and Potts [1]; Hall and Potts [2]; Chen and Vairaktarakis [3]; Chen and Pundoor [4]; Ji et al. [5]; $\mathrm{Li}$ and $\mathrm{Ou}$ [6]; Wang and Cheng [7]; Armstrong et al. 
[8]; Jiang and Li [9]; Jiang et al. [10]; Li et al. [11]; and Viergutz and Knust [12]). However, in some applications orders have arbitrary release dates. Hence, some related models involving release dates were also studied. Potts [13] was the first to study the model with single machine in which each order is delivered individually and immediately upon its completion. A heuristic with a worst-case ratio bound of $3 / 2$ was proposed to minimize the arrival time of the last order. After that, Hall and Shmoys [14] proposed a better heuristic for the model with a worst-case ratio bound of 4/3. Zdrzałka [15] provided a heuristic with a worst-case ratio bound of $3 / 2$ for a similar model with constraints that preemption is permitted and a sequence-independent setup time is incurred before processing a job. Gharbi and Haouari [16] studied a model with identical-parallel-machine configuration and proposed an exact branch-and-bound algorithm to solve the model. In addition, a polynomial-time approximation scheme was presented for this model by Mastrolilli [17]. Moreover, the single machine online model was also studied by Seiden [18], Hoogeveen and Vestjens [19], and van den Akker et al. [20], respectively. In recent years, batch delivery and delivery cost are involved in the models. Averbakh and Xue [21] and Averbakh [22] assumed that the orders are released online and batch delivered to the customer. To minimize the sum of the total flow time and total delivery cost, they proposed online algorithms for the single machine model with uncapacitated and capacitated deliveries, respectively. Fan [23] presented an approximation algorithm for the offline version of the model with uncapacitated deliveries proposed by Averbakh and Xue [21]. Lu et al. [24] studied an offline single machine model with capacitated deliveries that assumed that only one vehicle is available to deliver orders. They showed that the problem of minimizing the arrival time of the last order is strongly NP-hard and provided a 5/3-approximation algorithm. Liu and $\mathrm{Lu}$ [25] proposed an improved approximation algorithm for the model, which improved the performance ratio from $5 / 3$ to $3 / 2$. However, to the best of our knowledge, little attention is paid on the offline model with release dates in which the delivery batches are capacitated and delivery cost is considered in the objective functions. For more information, we refer the reader to Chen [26] for a comprehensive review on integrated production-distribution scheduling models.

The model we study in this paper can be viewed as an extension of production scheduling model with release dates. In production scheduling models, the following two functions are analogous to two widely used functions for measuring customer service: total completion time $C_{\text {total }}=\sum_{j \in N} C_{j}$ and maximum completion time $C_{\max }=\max _{j \in N} C_{j}$. In the following, we review some articles on single machine scheduling models with release dates. For ease of presentation, we adopt the well-known three-field notation $\alpha|\beta| \gamma$ introduced by Graham et al. [27], where $\alpha$ describes the machine configuration, $\beta$ specifies restrictions and constraints associated with the jobs, and $\gamma$ describes the objective function. Baker [28] showed that the preemptive model $1\left|r_{j}, p m t n\right| C_{\text {total }}$ is polynomially solvable, whereas Lenstra et al. [29] showed that the nonpreemptive model $1\left|r_{j}\right| C_{\text {total }}$ is NP-hard in the strong sense. In addition, the model $1\left|r_{j}, p m t n\right| C_{\max }$ is polynomially solvable. Lawler [30] pointed out that the nonpreemptive model $1\left|r_{j}\right| C_{\max }$ is also polynomially solvable. Since both production scheduling with release dates and batch delivery scheduling are considered, our model will be more complex. This leads us to be interested in the computational complexity of $P 1$ and $P 2$.

In this paper, we analyze the computational complexity of the problems and provide efficient algorithms for the problems, respectively. The paper is organized as follows. In Sections 2 and 3, we study the problems with different objective functions, respectively. In Section 4, we conclude the paper.

\section{P1: Minimizing $D_{\max }+T$}

In this section, we first introduce some optimality properties satisfied for problem $P 1$. Then, we provide a polynomialtime exact algorithm for it. We now present some preliminary results about the structure of an optimal solution of $P 1$ as follows.

Lemma 1. There exists an optimal solution for problem $P 1$ in which all of the following hold:

(1) The orders are delivered in the increasing sequence of their processing completion time.

(2) The orders are delivered in $z=\lceil n / b\rceil$ shipments, where $\lceil n / b\rceil$ is the smallest integer no less than $n / b$. The first shipment contains $n-(z-1) b$ orders and each of the remaining $z-1$ shipments contains $b$ orders.

(3) The departure time of each shipment is the time when all the orders in it complete processing.

The above optimality properties are straightforward; we omit the proofs.

Further, we define an Earliest Release Date first (ERD) rule. That is, a set of customer orders is in ERD order if the customer orders are sequenced in a nondecreasing order of their release dates, and the customer orders with equal release dates are sequenced in the arbitrary order.

Lemma 2. There exists an optimal solution for problem $P 1$ in which the customer orders are scheduled in ERD order.

Proof. By Lemma 1, the orders are delivered by $z=\lceil n / b\rceil$ vehicles in an optimal solution of problem $P 1$, which is independent of how the orders are scheduled on the machine. Hence, problem $P 1$ is reduced to minimizing $D_{\max }$. Because the delivery time of each shipment is assumed to be 0 , we have $D_{\max }=C_{\max }$ for a given solution, in which $C_{\max }$ is the maximum processing completion time of orders. As a result, the optimal solution of single machine makespan scheduling problem with release dates $1\left|r_{j}\right| C_{\max }$ is an optimal order processing schedule of problem $P 1$. Note that ERD rule provides an optimal solution for problem $1\left|r_{j}\right| C_{\max }$ [30]. Hence, ERD rule provides an optimal order processing schedule for problem $P 1$. 
Based on the results stated in Lemmas 1 and 2, an exact algorithm for problem $P 1$ is given as follows.

\section{Algorithm EA}

Step 1. Place all orders in a list in ERD order. Reindex the orders by their position in the list. Set current instant $t=r_{1}$.

Step 2. Choose the first unscheduled order $j$ of the list. Schedule it to process at instant $t^{\prime}=\max \left\{t, r_{j}\right\}$. Set $t=t^{\prime}+p_{j}$. Repeat Step 2 until all orders are scheduled.

Step 3. Deliver the orders such that they satisfy Lemma 1.

In algorithm EA, the majority of computation time is used for sorting orders in Step 1, which takes $O(n \log n)$ time. Hence, we have the following.

Theorem 3. Algorithm EA finds an optimal solution of problem $P 1$ in $O(n \log n)$ time.

\section{P2: Minimizing $D_{\text {total }}+T$}

When delivery cost of each shipment is ignored, that is, $f=0$, problem $P 2$ is reduced to problem $1\left|r_{j}\right| C_{\text {total }}$, which is known to be NP-hard in the strong sense [29]. Thus, problem $P 2$ is also NP-hard in the strong sense. To solve problem $P 2$, we first study an auxiliary problem $P_{\text {aux }}$. Everything else in problem $P_{\text {aux }}$ is the same as in problem $P 2$ except that preemption is allowed; that is, order processing can be preempted and resumed later. We first introduce an optimality property of $P_{\text {aux }}$ related to the Shortest Remaining Processing Time first (SRPT) rule, which prescribes to process at each instant $t$ the job with the smallest remaining processing time among all already released unfinished jobs, with some unambiguous rule for breaking ties. For example, the jobs can be indexed, and in case of a conflict between jobs with equal remaining processing times, the job with the smallest index would be given priority; such rule is called lexicographic [22]. According to this definition, there is exactly one SRPT order for a given set of jobs. Moreover, for two classical preemptive problems $1\left|r_{j}, p m t n\right| C_{\text {total }}$ and $1\left|r_{j}, p m t n\right| C_{\max }$, the SRPT rule provides an optimal solution [28]. Pruhs et al. [31] also presented the following result.

Lemma 4. In the SRPT job schedule, at any instant the number of processed jobs is not smaller than in any other job schedule.

According to Lemma 4, we obtain the following result satisfied for problem $P_{\text {aux }}$.

Lemma 5. There exists an optimal solution for problem $P_{\text {aux }}$ in which the orders are processed in the sequence ordered by SRPT rule on the machine.

Proof. Suppose that there exists an optimal solution in which the order processing schedule does not follow the SRPT rule. Denote the number of vehicles used in this solution by $k^{*}$. Let $\tau_{k}$ be the arrival time of vehicle $k$ and $n_{k}$ the number of the finished orders in time $\tau_{k}$ in this solution, $k=1, \ldots, k^{*}$.
Clearly, $n_{k^{*}}=n$. Then, the total arrival time of orders in this solution is equal to

$$
n_{1} \tau_{1}+\sum_{k=1}^{k^{*}-1}\left(n_{k+1}-n_{k}\right) \tau_{k+1}=\sum_{k=1}^{k^{*}-1} n_{k}\left(\tau_{k}-\tau_{k+1}\right)+n \tau_{k^{*}}
$$

By Lemma 4, at any instant $k$ the number of processed orders $n_{k}$ in the SRPT order processing schedule is the biggest one in all order processing schedules. In addition, $\tau_{k}-\tau_{k+1}<$ 0 , for $k=1, \ldots, k^{*}-1$. Hence, we can rearrange the jobs in the SRPT order without increasing $\sum_{k=1}^{k^{*}-1} n_{k}\left(\tau_{k}-\tau_{k+1}\right)$. Also, because the SRPT rule provides an optimal solution for $1\left|r_{j}, p m t n\right| C_{\max }$, the SRPT order processing schedule is the one in which $\tau_{k^{*}}$ is minimized. Therefore, we can rearrange the customer orders in the SRPT order without increasing the objective value.

Without loss of generality, we assume that the customer orders are indexed in SRPT order so that $C_{1} \leq \cdots \leq C_{n}$. The following dynamic programming algorithm finds an optimal solution for $P_{\text {aux }}$.

Algorithm DP. Define value function $V(j)=$ minimum total cost contribution of a solution for the first $j$ orders $\{1, \ldots, j\}$.

$$
\begin{aligned}
& \text { Initial condition: } V(0)=0 \text {. } \\
& \text { Recursive relation: for } j=1, \ldots, n, \\
& \qquad V(j)=\min \left\{V(j-h)+h \cdot C_{j}+f \mid h\right. \\
& \quad=1, \ldots, \min (b, j)\} .
\end{aligned}
$$

Optimal solution value: it is $V(n)$.

Theorem 6. Algorithm DP finds an optimal solution of problem $P_{\text {aux }}$ in $O(n \log n+n b)$ time.

Proof. By Lemma 5, in a solution for the first $j$ order $\{1, \ldots, j\}$, where the production schedule follows SRPT rule, the departure time of the last vehicle is always $C_{j}$. This implies that the delivery time of all the orders in the last vehicle is $C_{j}$. In the recursive relation, the value function is computed by trying every possible size of the last vehicle. Given that the size of the last vehicle is $h$, the total cost contributed by the last vehicle is $h \cdot C_{j}+f$, where $h \cdot C_{j}$ is the contribution to the total delivery time and $f$ is the distribution cost. This proves the correctness of the recursive relation and hence the optimality of the algorithm. There are $n$ states in this DP, and it takes no more than $O(b)$ time to calculate the value for each state. Sorting the orders by SRPT rule takes $O(n \log n)$ time. Therefore, the overall time complexity of DP is bounded by $O(n \log n+n b)$.

Corollary 7. The value $V(n)$ generated by algorithm DP is a lower bound of the optimal objective value of $P 2$.

Proof. Because the optimal solution of $P 2$ is a feasible solution of $P_{\text {aux }}$, the optimal objective value of $P_{\text {aux }}$ is no larger than that of $P 2$. By Theorem 6 , the solution obtained 
by algorithm DP is an optimal one for problem $P_{\text {aux }}$. Hence, the value $V(n)$ generated by algorithm DP is a lower bound of the optimal objective value of $P 2$.

In the remainder of this section, we present a polynomialtime heuristic for problem $P 2$ and analyze its worst-case performance.

\section{Heuristic HA}

Step 1. Run algorithm DP to obtain a basic solution $S_{\text {aux }}^{*}$. Denote the number of vehicles used in this solution by $k^{*}$. Let $C_{\text {aux }}(j)$ be denoted as the processing completion time of order $j$, for $j \in N$. Place all orders in a list sorted by an increasing order of their processing completion times $C_{\text {aux }}(j)$ in this basic solution. Reindex the orders by their position in the list. Set current instant $t=r_{1}$.

Step 2. Choose the first unscheduled order $j$ of the list. Schedule it to process at instant $t^{\prime}=\max \left\{t, r_{j}\right\}$. Set $t=t^{\prime}+p_{j}$. Repeat Step 2 until all orders are scheduled.

Step 3. Use $k^{*}$ vehicles to deliver orders. Each vehicle delivers the same orders as in $S_{\text {aux }}^{*}$. The departure time of each vehicle is the time when all the orders in it complete processing. Denote the resulting solution by $S^{*}$.

Clearly, the time complexity of heuristic HA is $\mathrm{O}(n \log n+$ $n b)$ because the majority of its computation time is to run algorithm DP. Next, we analyze its worst-case performance. Denote the optimal objective value of problems $P 2$ and $P_{\text {aux }}$ by $F_{P 2}^{*}$ and $F_{\text {aux }}^{*}$, respectively. Let $F_{P 2}\left(S^{*}\right)$ be denoted as the contribution of solution $S^{*}$ to the objective value of problem P2.

Theorem 8. $F_{P 2}\left(S^{*}\right) \leq 2 F_{P 2}^{*}$; that is, the worst-case ratio of heuristic $H A$ is bounded by 2 .

Proof. In $S^{*}$, let $\bar{k}$ be the order in vehicle $B_{k}$ with largest processing completion time $C_{j}, k=1, \ldots, k^{*}$. Clearly, $C_{\bar{k}}=$ $D_{B_{k}}$. Because there is no idle time between instant $\max _{j \in B_{k}} r_{j}$ and $C_{\bar{k}}$, we have

$$
C_{\bar{k}}=D_{B_{k}} \leq \max _{j \in B_{k}} r_{j}+\sum_{h=1}^{k} \sum_{j \in B_{h}} p_{j}
$$

Denote the arrival time of order $j$ in $S_{\text {aux }}^{*}$ by $D_{\text {aux }}(j)$, for $j \in N$. Let $D_{\text {aux }}\left(B_{k}\right)$ be denoted as the arrival time of vehicle $B_{k}$ in $S_{\text {aux }}^{*}, k=1, \ldots, k^{*}$. For each order $j$ in vehicle $B_{k}$, we have $r_{j} \leq C_{\text {aux }}(j) \leq C_{\text {aux }}(\bar{k})$. This implies that $\max _{j \in B_{k}} r_{j} \leq$ $C_{\text {aux }}(\bar{k})$. In addition, $\sum_{h=1}^{k} \sum_{j \in B_{h}} p_{j} \leq C_{\text {aux }}(\bar{k})$. Hence, we have $C_{\bar{k}} \leq 2 C_{\text {aux }}(\bar{k})$. Consequently, $\left|B_{k}\right| D_{B_{k}} \leq 2\left|B_{k}\right| D_{\text {aux }}\left(B_{k}\right)$. Then,

$$
\sum_{j \in N} D_{j}=\sum_{k=1}^{k^{*}}\left|B_{k}\right| D_{B_{k}} \leq 2 \sum_{k=1}^{k^{*}}\left|B_{k}\right| D_{\text {aux }}\left(B_{k}\right) .
$$

Since the numbers of vehicles used in $S_{\text {aux }}^{*}$ and $S^{*}$ are equal, the distribution cost of these solutions is also identical.
Hence, $F_{P 2}\left(S^{*}\right) \leq 2 F_{\text {aux }}^{*}$. By Corollary 7 , we have $F_{\text {aux }}^{*} \leq F_{P 2}^{*}$. Hence, $F_{P 2}\left(S^{*}\right) \leq 2 F_{P 2}^{*}$.

\section{Conclusions}

In this paper, we have studied two variations of the integrated scheduling of production and distribution model with release dates and capacitated deliveries, each of which has a different objective function. The first variation is to minimize the arrival time of the last order plus total distribution cost; we first presented some optimality properties satisfied for the variation. Adopting these optimality properties, we proposed a polynomial-time exact algorithm which implies that this problem is easy. The second variation is to minimize total arrival time of the orders plus total distribution cost; due to its NP-hard property, we developed a heuristic for it. In this heuristic, we first construct an auxiliary problem which is the problem with preemptions and develop a dynamic programming algorithm for the auxiliary problem. Then we propose the heuristic based on the solution obtained by the dynamic programming algorithm. Finally, we analyze its worse-case performance and show that its worst-case ratio is bounded by 2 .

For future research, it will be worth extending the existing model to parallel machines and developing effective and efficient algorithms.

\section{Competing Interests}

The authors declare that there are no competing interests regarding the publication of this paper.

\section{Acknowledgments}

This research was supported in part by the National Natural Science Foundation of China (71501051), the Humanities and Social Sciences Research Foundation of Ministry of Education of China (13YJC630239), and the Foundation for Distinguished Young Teachers in Higher Education of Guangdong Province (YQ201403).

\section{References}

[1] N. G. Hall and C. N. Potts, "Supply chain scheduling: batching and delivery," Operations Research, vol. 51, no. 4, pp. 566-584, 2003.

[2] N. G. Hall and C. N. Potts, "The coordination of scheduling and batch deliveries," Annals of Operations Research, vol. 135, no. 1, pp. 41-64, 2005.

[3] Z.-L. Chen and G. L. Vairaktarakis, "Integrated scheduling of production and distribution operations," Management Science, vol. 51, no. 4, pp. 614-628, 2005.

[4] Z.-L. Chen and G. Pundoor, "Order assignment and scheduling in a supply chain," Operations Research, vol. 54, no. 3, pp. 555$572,2006$.

[5] M. Ji, Y. He, and T. C. E. Cheng, "Batch delivery scheduling with batch delivery cost on a single machine," European Journal of Operational Research, vol. 176, no. 2, pp. 745-755, 2007. 
[6] C.-L. Li and J. Ou, "Coordinated scheduling of customer orders with decentralized machine locations," IIE Transactions (Institute of Industrial Engineers), vol. 39, no. 9, pp. 899-909, 2007.

[7] X. Wang and T. C. E. Cheng, "Machine scheduling with an availability constraint and job delivery coordination," Naval Research Logistics, vol. 54, no. 1, pp. 11-20, 2007.

[8] R. Armstrong, S. Gao, and L. Lei, "A zero-inventory production and distribution problem with a fixed customer sequence," Annals of Operations Research, vol. 159, pp. 395-414, 2008.

[9] D. K. Jiang and B. Li, "Supply chain scheduling based on hybrid taboo search algorithm," Journal of Mechanical Engineering, vol. 47, no. 20, pp. 53-59, 2011.

[10] D.-K. Jiang, B. Li, and J.-Y. Tan, "Integrated optimization approach for order assignment and scheduling problem," Control and Decision, vol. 28, no. 2, pp. 217-222, 2013.

[11] S. Li, X. Zhong, H. Li, and S. Li, "Batch delivery scheduling with multiple decentralized manufacturers," Mathematical Problems in Engineering, vol. 2014, Article ID 321513, 7 pages, 2014.

[12] C. Viergutz and S. Knust, "Integrated production and distribution scheduling with lifespan constraints," Annals of Operations Research, vol. 213, no. 1, pp. 293-318, 2014.

[13] C. N. Potts, "Analysis of a heuristic for one machine sequencing with release dates and delivery times," Operations Research, vol. 28, no. 6, pp. 1436-1441, 1980.

[14] L. A. Hall and D. B. Shmoys, "Jackson's rule for single-machine scheduling: making a good heuristic better," Mathematics of Operations Research, vol. 17, no. 1, pp. 22-35, 1992.

[15] S. Zdrzałka, "Preemptive scheduling with release dates, delivery times and sequence independent setup times," European Journal of Operational Research, vol. 76, no. 1, pp. 60-71, 1994.

[16] A. Gharbi and M. Haouari, "Minimizing makespan on parallel machines subject to release dates and delivery times," Journal of Scheduling, vol. 5, no. 4, pp. 329-355, 2002.

[17] M. Mastrolilli, "Efficient approximation schemes for scheduling problems with release dates and delivery times," Journal of Scheduling, vol. 6, no. 6, pp. 521-531, 2003.

[18] S. Seiden, "Randomized online scheduling with delivery times," Journal of Combinatorial Optimization, vol. 3, no. 4, pp. 399416, 1999.

[19] J. A. Hoogeveen and A. P. Vestjens, "A best possible deterministic on-line algorithm for minimizing maximum delivery time on a single machine," SIAM Journal on Discrete Mathematics, vol. 13, no. 1, pp. 56-63, 2000.

[20] M. van den Akker, H. Hoogeveen, and N. Vakhania, "Restarts can help in the on-line minimization of the maximum delivery time on a single machine," Journal of Scheduling, vol. 3, no. 6, pp. 333-341, 2000.

[21] I. Averbakh and Z. Xue, "On-line supply chain scheduling problems with preemption," European Journal of Operational Research, vol. 181, no. 1, pp. 500-504, 2007.

[22] I. Averbakh, "On-line integrated production-distribution scheduling problems with capacitated deliveries," European Journal of Operational Research, vol. 200, no. 2, pp. 377-384, 2010.

[23] J. Fan, "Supply chain scheduling with job's release times on a single machine," Journal of Systems Science and Mathematical Sciences, vol. 31, no. 11, pp. 1439-1443, 2011.

[24] L. Lu, J. Yuan, and L. Zhang, "Single machine scheduling with release dates and job delivery to minimize the makespan," Theoretical Computer Science, vol. 393, no. 1-3, pp. 102-108, 2008.
[25] P. Liu and X. Lu, "An improved approximation algorithm for single machine scheduling with job delivery," Theoretical Computer Science, vol. 412, no. 3, pp. 270-274, 2011.

[26] Z.-L. Chen, "Integrated production and outbound distribution scheduling: review and extensions," Operations Research, vol. 58, no. 1, pp. 130-148, 2010.

[27] R. L. Graham, E. L. Lawler, J. K. Lenstra, and A. H. G. Rinnooy Kan, "Optimization and approximation in deterministic sequencing and scheduling: a survey," Annals of Discrete Mathematics, vol. 4, pp. 287-326, 1979.

[28] K. R. Baker, Introduction to Sequencing and Scheduling, John Wiley \& Sons, New York, NY, USA, 1974.

[29] J. K. Lenstra, A. H. R. Rinnooy Kan, and P. Brucker, "Complexity of machine scheduling problems," Annals of Discrete Mathematics, vol. 1, pp. 343-362, 1977.

[30] E. L. Lawler, "Optimal sequencing of a single machine subject to precedence constraints," Management Science, vol. 19, no. 5, pp. 544-546, 1973.

[31] K. Pruhs, J. Sgall, and E. Torng, "On-line scheduling," in Handbook of Scheduling: Algorithms, Models, and Performance Analysis, J. Y.-T. Leung, Ed., CRC Press, New York, NY, USA, 2004. 


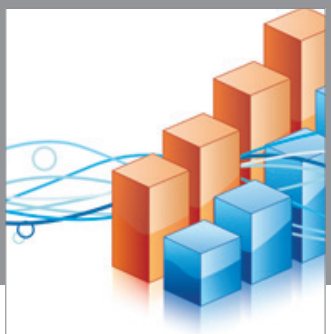

Advances in

Operations Research

vatem alat4



\section{The Scientific} World Journal
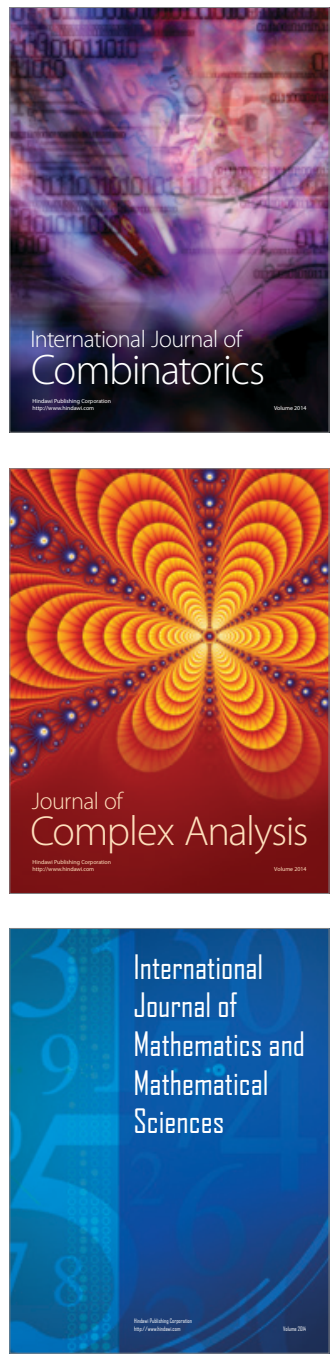
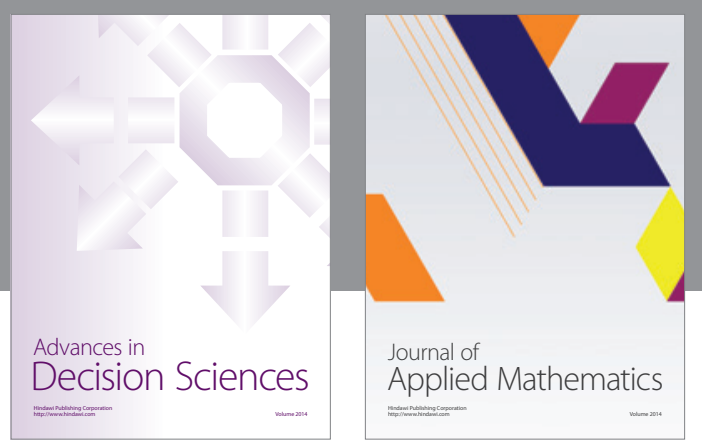

Algebra

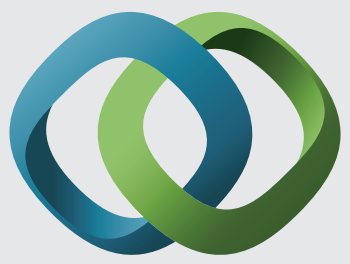

\section{Hindawi}

Submit your manuscripts at

http://www.hindawi.com
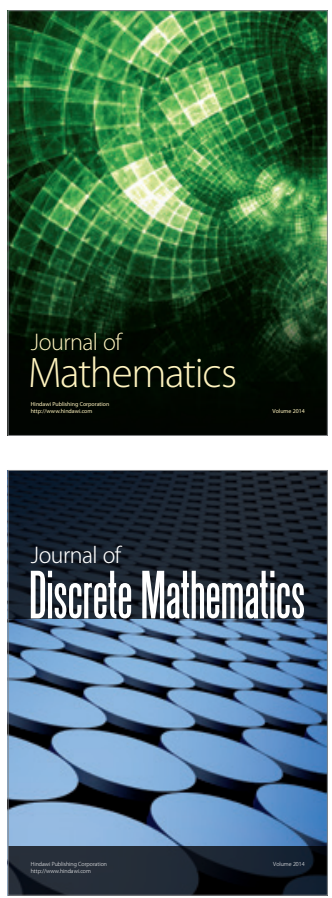

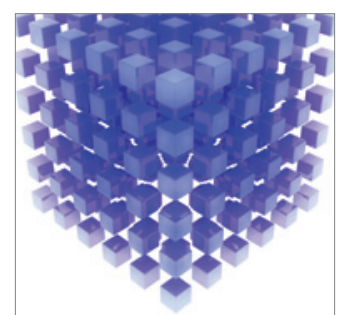

Mathematical Problems in Engineering
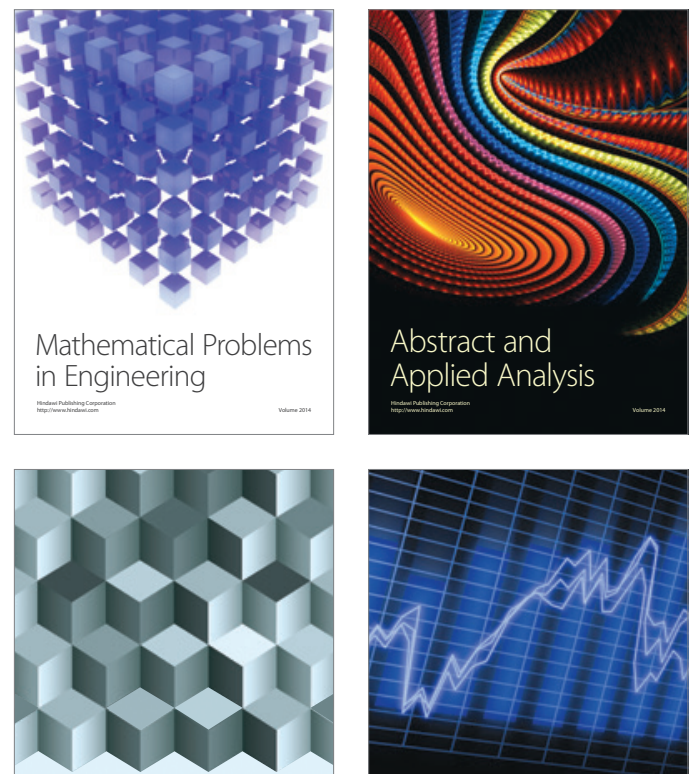

Journal of

Function Spaces

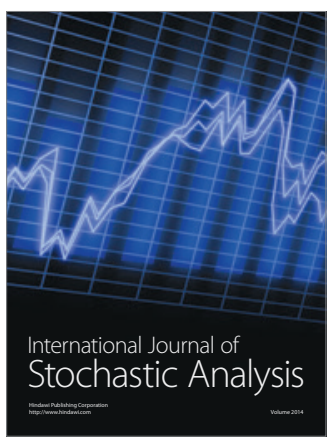


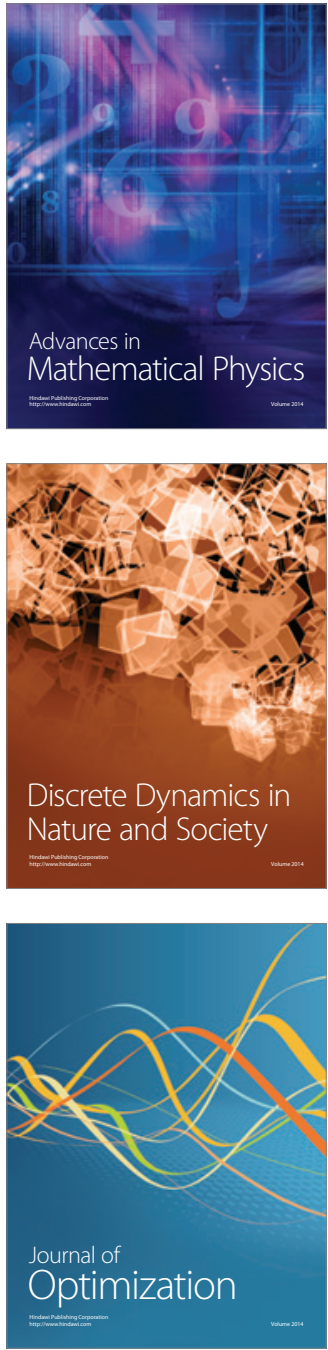